\title{
Passivation of Silicon Surface by Laser Rapid Heating
}

\author{
H. Abe ${ }^{1}$, C. Akiyama ${ }^{1}$, M. Hasumi ${ }^{1}$, T. Sameshima ${ }^{1}$, T. Mizuno ${ }^{2}$, and N. Sano ${ }^{3}$ \\ ${ }^{*}$ Tokyo University of Agriculture and Technology, 2-24-16, Naka-cho, Koganei, 184-8588, Japan \\ E-mail: tsamesim@cc.tuat.ac.jp \\ *22 Kanagawa University, 2946 Tsuchiya, Hiratsuka 259-1293, Japan \\ *33 Aurea Works Corporation, 75-1, Ono-cho, Tsurumi-ku, Yokohama 230-0046, Japan
}

\begin{abstract}
We report rapid laser-induced passivation of the silicon surface. When the top surfaces of n-type 500- $\mu \mathrm{m}$-thick silicon substrates with surfaces coated with $100 \mathrm{~nm}$ thermally grown $\mathrm{SiO}_{2}$ layers (initial samples) were irradiated with Ar plasma at $50 \mathrm{~W}$ for $120 \mathrm{~s}$, the 635-nm-light induced minority carrier effective lifetime $\tau_{\text {eff }}$ decreased from $1.7 \times 10^{-3}$ (initial) to $1.7 \times 10^{-5} \mathrm{~s}$ because Ar plasma caused substantial carrier recombination defect states at the silicon surfaces. $\tau_{\text {eff }}$ was markedly increased to $1.7 \times 10^{-3}$ s by 940-nm-semiconductor laser irradiation at $3.57 \times 10^{4} \mathrm{~W} / \mathrm{cm}^{2}$ for $4 \mathrm{~ms}$. Laser heating effectively decreased the density of plasma induced carrier recombination defect. However, laser heating of the initial sample at $4.0 \times 10^{4} \mathrm{~W} / \mathrm{cm}^{2}$ decreased $\tau_{\text {eff }}$ from $2.2 \times 10^{-3}$ (initial) to $1.7 \times 10^{-4} \mathrm{~s}$. Additional laser heating at $3.70 \times 10^{4} \mathrm{~W} / \mathrm{cm}^{2}$ increased $\tau_{\text {eff }}$ to $3.9 \times 10^{-4} \mathrm{~s}$-and it partially cured laser induced carrier recombination defects.

DOI: $10.2961 / \mathrm{j} 1 \mathrm{mn} .2014 .02 .0012$
\end{abstract}

Keywords: minority carrier effective lifetime, laser heating, semiconductor laser, microwave absorption, carrier recombination

\section{Introduction}

Laser heating is an attractive method for activating silicon semiconductor implanted with dopant atoms [1-6]. A high activation ratio and no marked impurity diffusion are achieved by laser-induced rapid heating. No substrate heating is necessary because laser heating energy effectively concentrates in the surface region during short irradiation time. It is important to reduce the thermal budget for fabricating semiconductor devices at a low cost. A laser heating is also attractive for annealing of semiconductor surface region. Defects located at the surface region will be reduced and thermally stable state will be achieved by laser heating with no substrate heating. However, we recently found that laser heating decreased the minority carrier effective lifetime $\tau_{\text {eff }}$ of silicon substrate [7]. Decrease in $\tau_{\text {eff }}$ will be a serious problem for application of laser heating to fabrication of high efficiency solar cells and high sensitivity photo sensors because of low light sensitivity.

In this paper, we discuss laser-induced passivation of silicon surface by $940-\mathrm{nm}$ semiconductor laser heating. We demonstrate that laser heating effectively increases $\tau_{\text {eff, }}$ which was beforehand decreased by Ar plasma irradiation. The laser power condition for increase in $\tau_{\text {eff }}$ is limited. We also demonstrate decrease in $\tau_{\text {eff }}$ by laser irradiation at a high power intensity. We discuss physics of laser heating to annihilate or create carrier recombination defects.

\section{Experimental}

$12 \Omega \mathrm{cm}$ n-type 500- $\mu \mathrm{m}$-thick silicon substrates were prepared. The top and rear surfaces were coated with 100 nm-thick thermally grown $\mathrm{SiO}_{2}$ layers formed in $1100^{\circ} \mathrm{C}$ wet oxygen atmosphere. The silicon samples were irradiated with 940-nm infrared semiconductor laser. Figure 1 shows a schematic apparatus of laser heating. The laser beam with a maximum power of $25 \mathrm{~W}$ was introduced using an optical fiber. The optical fiber and optics with a lens were mounted on an $X-Y$ mobile stage. The laser beam was focused by the lens to a spot with a Gaussian intensity distribution and a diameter of $200 \mu \mathrm{m}$ at full width and maximum half at the sample surface. Samples were placed on a 1-mm-thick quartz glass plate in a direction normal to the laser beam. The laser beam was moved in the $Y$-direction at $5 \mathrm{~cm} / \mathrm{s}$ at power intensities ranging from $2.67 \times 10^{4}$ to $4.46 \times 10^{4} \mathrm{~W} / \mathrm{cm}^{2}$ at sample surface. Irradiation duration was $4 \mathrm{~ms}$. The laser beam was stepwise moved in the $X$ direction at a step of $100 \mu \mathrm{m}$. The top surface of the sample with an area of $4 \times 5 \mathrm{~cm}^{2}$ was completely heated by laser irradiation with a overlapping ratio of $50 \%$ for $4 \mathrm{~ms}$. Heat flow calculation using a numerical finite-element program resulted in heating to $1060 \mathrm{~K}$ at the top surface for conditions of a laser intensity of $4.46 \times 10^{4} \mathrm{~W} / \mathrm{cm}^{2}$ and a light reflection loss of $17 \%$ at $940 \mathrm{~nm}$.

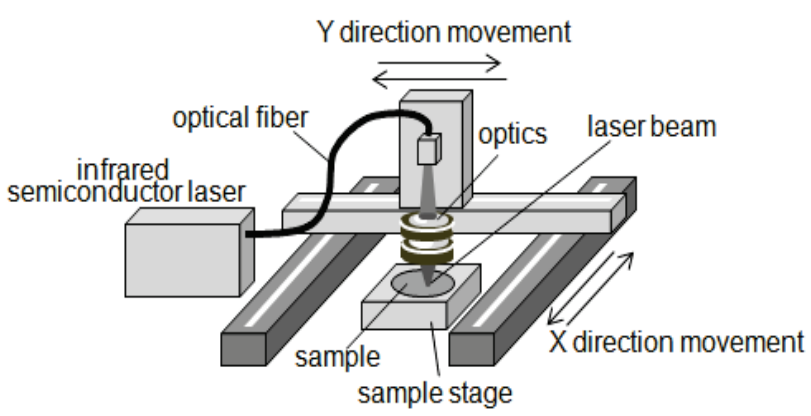

Fig. 1. Schematic apparatus of infrared semiconductor laser heating. 
The top surfaces of the samples were irradiated by 13.56 MHz radio-frequency (RF)-capacitance-coupled Ar plasma at $50 \mathrm{~W}$ and $1.0 \mathrm{~Pa}$ for $120 \mathrm{~s}$ [8]. A sample was placed on a metal plate electrically grounded in a chamber facing a metal electrode applied at RF voltage. Ar gas was introduced at $50 \mathrm{sccm}$ under evacuation using a turbomolecular pump.

Figure 2 shows a schematic of the $9.35 \mathrm{GHz}$ microwave transmittance measurement system with waveguide tubes, which had a narrow gap for placing a sample wafer. Continuous wave $(\mathrm{CW}) 635 \mathrm{~nm}$ laser diode (LD) lights at 1.5 $\mathrm{mW} / \mathrm{cm}^{2}$ were introduced in the waveguide tube, as shown in Fig. 2. The microwave which transmitted samples was rectified using a high-speed diode and analyzed to obtain $\tau_{\text {eff }}[9,10]$. We constructed a finite-element numerical calculation program including theories of carrier generation associated with optical absorption coefficients, carrier diffusion, and annihilation to estimate the carrier recombination defect states from experimental $\tau_{\text {eff }}$ (top) and $\tau_{\text {eff }}$ (rear) obtained by light illumination to the top and rear surfaces, respectively $[11,12]$.

After the microwave transmittance measurement, the $\mathrm{SiO}_{2}$ layers at the rear surface were removed by $5 \%$ hydrofluoric acid. Al metal electrodes were formed at the top and rear surfaces by the vacuum evaporation. The capacitance response at $1 \mathrm{MHz}$ alternative voltage with an amplitude of $10 \mathrm{mV}$ as a function of the bias voltage $(\mathrm{C}-\mathrm{V})$ was measured.

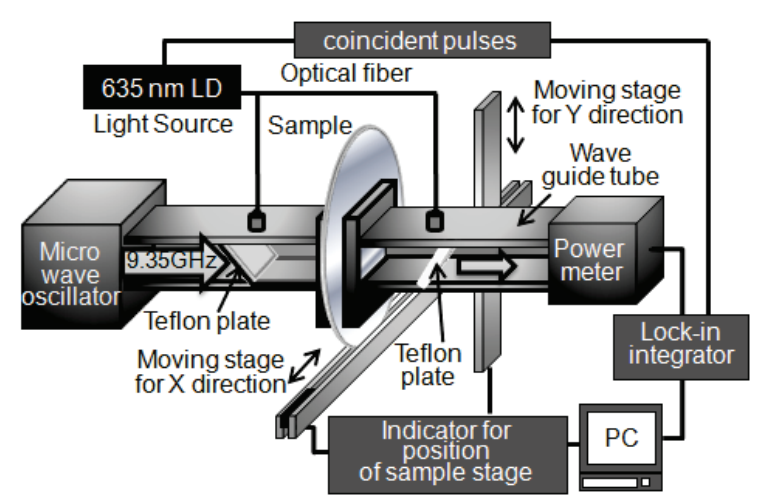

Fig. 2. Schematic apparatus of the $9.35 \mathrm{GHz}$ microwave transmittance measurement system

\section{Results and discussion}

Figures 3 shows $\tau_{\text {eff }}$ (top) (open circles) and $\tau_{\text {eff }}$ (rear) (solid circles) as a function of laser power intensity for laser irradiation applied to the initial (a) and Ar plasma irradiated samples (b). $\tau_{\text {eff }}$ (top) and $\tau_{\text {eff }}$ (rear) had a high value of $1.5 \times 10^{-3} \mathrm{~s}$ for the initial sample, as shown by the arrow in Fig. 3(a). This means that the initial sample had a high quality crystalline bulk and the surfaces well passivated with thermally grown $\mathrm{SiO}_{2}$ layers. The samples were irradiated with 940 -nm-laser light with stepwise increasing its power intensity. $\tau_{\text {eff }}$ (top) and $\tau_{\text {eff }}$ (rear) were decreased by irradiation above $4.00 \times 10^{4} \mathrm{~W} / \mathrm{cm}^{2}$. They decreased to $2.5 \times 10^{-6}$ and $1.4 \times 10^{-4} \mathrm{~s}$ at $4.46 \times 10^{4} \mathrm{~W} / \mathrm{cm}^{2}$ as shown in Fig.

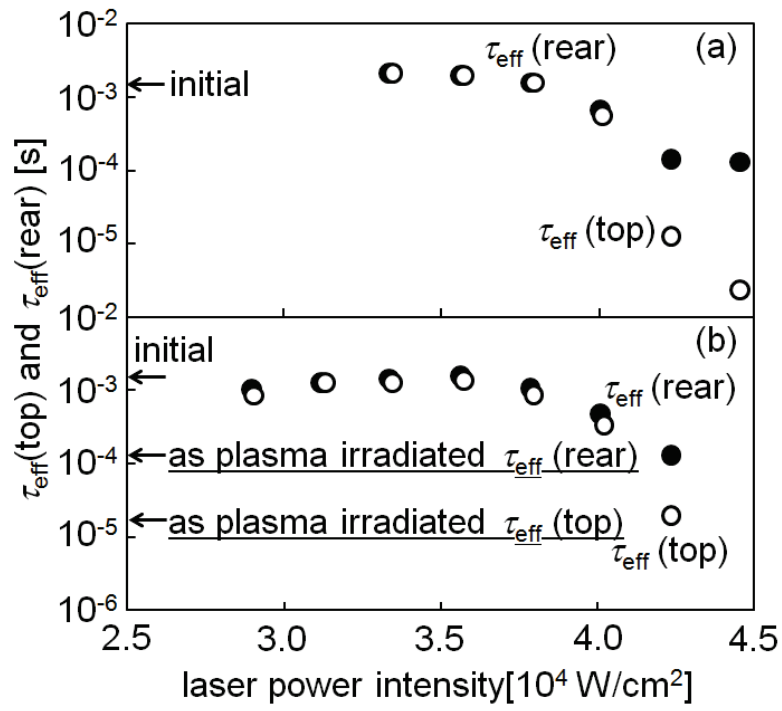

Fig. 3. $\tau_{\text {eff }}$ (top) (open circles) and $\tau_{\text {eff }}$ (rear) (solid circles) as a function of laser power intensity for laser irradiation applied to the initial (a) and Ar plasma irradiated samples (b).

3 (a). Those results mean that a high density of carrier recombination defect sites was generated at the surface region by infrared laser heating with a high power. The defect sites caused by laser irradiation effectively annihilate photo-induced carriers generated by 635 -nm-light illuminations to the top surface. $\tau_{\text {eff }}$ (top) was therefore very low. In contrast, photo-induced carriers generated by 635 -nm-light illumination to the rear surface traveled across the 500- $\mu \mathrm{m}$ thick substrate from the rear to the top surfaces and then they were annihilated by the recombination defect sites. Therefore, they were alive long during diffusion in the substrate and $\tau_{\text {eff }}$ (rear) was high [7].

When Ar plasma was applied to the initial sample, $\tau_{\text {eff }}$ (top) and $\tau_{\text {eff }}$ (rear) decreased from the initial value of $1.7 \times 10^{-3} \mathrm{~s}$ to $1.7 \times 10^{-5}$ and $1.4 \times 10^{-4} \mathrm{~s}$, as shown by three arrows in Fig. 3(b). The plasma irradiated sample was heated by laser with stepwise increasing its power intensity. $\tau_{\text {eff }}$ (top) and $\tau_{\text {eff }}$ (rear) increased to $1.7 \times 10^{-3} \mathrm{~s}$, almost the same as that of the initial sample, as the laser power intensity increased from $2.67 \times 10^{4}$ up to $3.57 \times 10^{4} \mathrm{~W} / \mathrm{cm}^{2}$. Laser heating cured the defect states caused by the Ar plasma irradiation. However, $\tau_{\text {eff }}$ (top) and $\tau_{\text {eff }}$ (rear) were decreased again by laser irradiation above $4.0 \times 10^{4} \mathrm{~W} / \mathrm{cm}^{2}$ for the plasma irradiated sample similar to the case of the initial sample. The results of Fig. 3 show that laser heating plays different roles of defect reduction and defect generation, which depended on the laser power intensity.

We then prepared the samples by two-step 940-nmlaser irradiation. For the first time, the initial samples were heated with $940-n m$-laser light at $4.00 \times 10^{4}$ and $4.40 \times 10^{4}$ $\mathrm{W} / \mathrm{cm}^{2}$. Then the samples were irradiated again by $940-\mathrm{nm}-$ laser light with stepwise increasing the power intensity. Figure 4 shows $\tau_{\text {eff }}$ (top) (open circles and squares) and $\tau_{\text {eff }}$ (rear) (solid circles and squares) as a function of laser power intensity at second irradiation. The first laser irradiation at $4.00 \times 10^{4} \mathrm{~W} / \mathrm{cm}^{2}$ decreased $\tau_{\text {eff }}$ (top) and $\tau_{\text {eff }}$ (rear) 


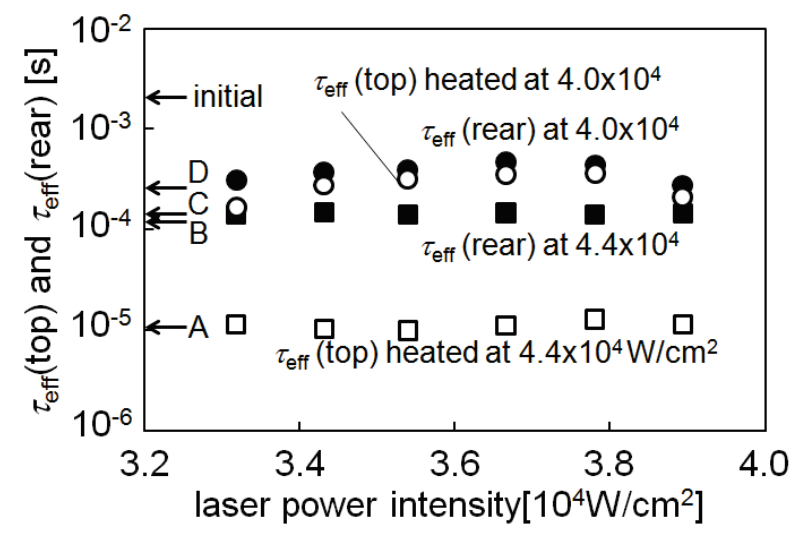

Fig. 4. $\tau_{\text {eff }}$ (top) (open marks) and $\tau_{\text {eff }}$ (rear) (solid marks) as a function of laser power intensity at second irradiation. Arrows A and B present $\tau_{\text {eff }}$ (top) and $\tau_{\text {eff }}$ (rear), for sample laser irradiated at $4.4 \times 10^{4} \mathrm{~W} / \mathrm{cm}^{2}$. Arrows C and D present $\tau_{\text {eff }}$ (top) and $\tau_{\text {eff }}$ (rear), for sample laser irradiated at $4.0 \times 10^{4} \mathrm{~W} / \mathrm{cm}^{2}$.

of $1.7 \times 10^{-4}$ and $2.9 \times 10^{-4} \mathrm{~s}$, as shown by arrows $\mathrm{C}$ and $\mathrm{D}$ in Fig. 4. They were increased to $3.9 \times 10^{-4}$ and $5.2 \times 10^{-4} \mathrm{~s}$ by second laser irradiation up to $3.70 \times 10^{4} \mathrm{~W} / \mathrm{cm}^{2}$. This indicates that the density of carrier recombination defect states was decreased by second laser irradiation. On the other hand, when the samples were irradiated with laser at $4.40 \times 10^{4} \mathrm{~W} / \mathrm{cm}^{2}, \tau_{\text {eff }}$ (top) and $\tau_{\text {eff }}$ (rear) were decreased to $1.1 \times 10^{-5}$ and $1.5 \times 10^{-4} \mathrm{~s}$, as shown by arrows $\mathrm{A}$ and $\mathrm{B}$, respectively, as shown in Fig. 4. They were hardly changed by second laser irradiation between $3.30 \times 10^{4}$ and $3.90 \times 10^{4}$ $\mathrm{W} / \mathrm{cm}^{2}$. Defect states formed by high power laser irradiation were not cured by second laser irradiation.

Figure 5 shows $\mathrm{C}-\mathrm{V}$ characteristics for the initial sample, laser irradiated samples at $4.00 \times 10^{4}$, and $4.40 \times 10^{4}$ $\mathrm{W} / \mathrm{cm}^{2}$, and two step laser irradiated samples at $4.0 \times 10^{4}$ followed by $3.70 \times 10^{4} \mathrm{~W} / \mathrm{cm}^{2}$, and at $4.40 \times 10^{4} \mathrm{~W} / \mathrm{cm}^{2}$ followed by $3.70 \times 10^{4} \mathrm{~W} / \mathrm{cm}^{2}$ (a) and the plasma irradiated sample and sample plasma irradiated followed by laser irradiated at $3.57 \times 10^{4} \mathrm{~W} / \mathrm{cm}^{2}$ (b). Sharp change in capacitance was observed for the initial sample. It means that the sample had a low density of interface traps. Capacitance change became gentle in the cases of laser irradiation at $4.0 \times 10^{4}$ and $4.4 \times 10^{4} \mathrm{~W} / \mathrm{cm}^{2}$ because of generation of interface trap states. Capacitance change became sharp in the case of two step laser irradiation at $4.0 \times 10^{4}$ followed by $3.7 \times 10^{4} \mathrm{~W} / \mathrm{cm}^{2}$, while the change in capacitance kept genthe in the case of laser irradiation at $4.4 \times 10^{4}$ followed by $3.7 \times 10^{4} \mathrm{~W} / \mathrm{cm}^{2}$, as shown in Fig. 5(a). Ar plasma treatment made capacitance change very gentle. Ar plasma irradiation caused substantial interface traps. Subsequent laser irradiation at $3.57 \times 10^{4} \mathrm{~W} / \mathrm{cm}^{2}$ made capacitance change sharp similar to that of the initial sample.

The density of surface carrier recombination defect states $D_{\mathrm{r}}$ and the density of interface traps $D_{\text {it }}$ at the mid gap were estimated from experimental $\tau_{\text {eff }}$ (top) and $\tau_{\text {eff }}$ (rear), and C-V characteristics, as shown in Figs. 3-5 [13]. Figure 6 shows $D_{\text {r }}$ (a) and $D_{\text {it }}$ (b) for samples shown by their $\mathrm{C}-\mathrm{V}$ curves in Fig. 5. $D_{\mathrm{r}}$ and $D_{\text {it }}$ were low of $7.6 \times 10^{9}$ $\mathrm{cm}^{-2}$ and $5.9 \times 10^{9} \mathrm{~cm}^{-2} \mathrm{eV}^{-1}$ for the initial sample. They increased to $3.1 \times 10^{12} \mathrm{~cm}^{-2}$ and $3.8 \times 10^{11} \mathrm{~cm}^{-2} \mathrm{eV}^{-1}$ for the

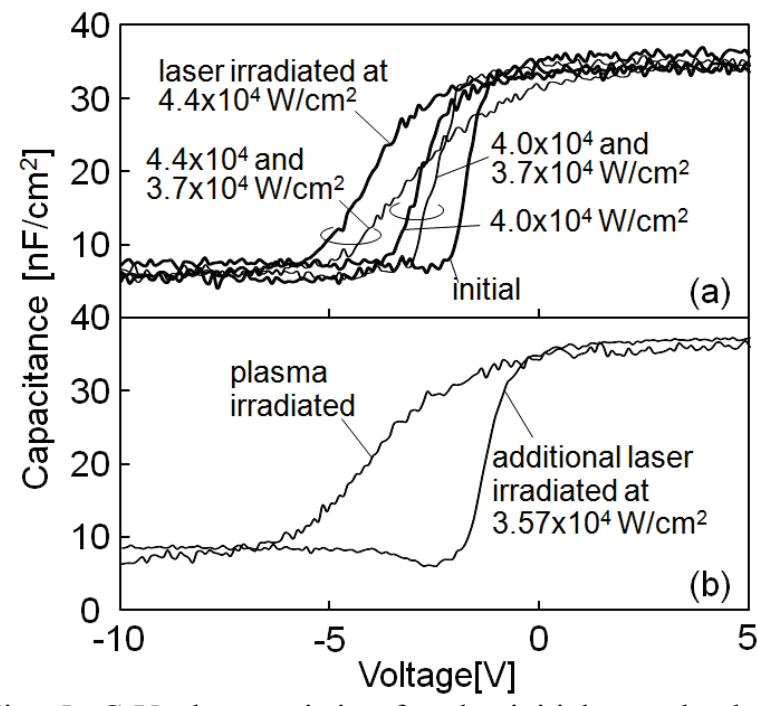

Fig. 5. C-V characteristics for the initial sample, laser irradiated samples at $4.0 \times 10^{4}$ and $4.4 \times 10^{4} \mathrm{~W} / \mathrm{cm}^{2}$, and two step laser irradiated samples at $4.0 \times 10^{4}$ followed by $3.7 \times 10^{4} \mathrm{~W} / \mathrm{cm}^{2}$, and at $4.4 \times 10^{4}$ followed by $3.7 \times 10^{4}$ $\mathrm{W} / \mathrm{cm}^{2}$ (a) and the plasma irradiated sample and sample plasma irradiated followed by laser irradiated at $3.57 \times 10^{4}$ $\mathrm{W} / \mathrm{cm}^{2}$ (b).

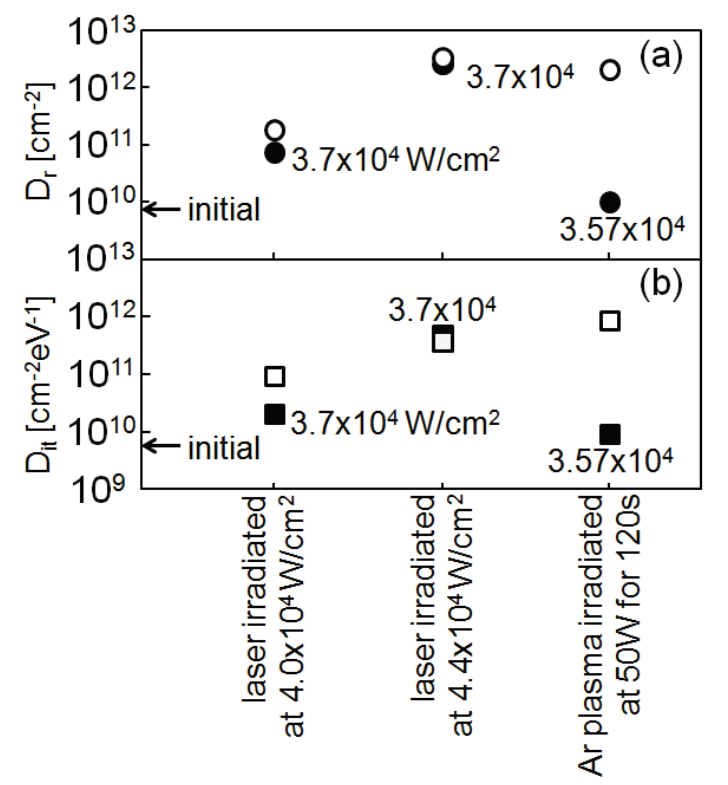

Fig. 6. $D_{\mathrm{r}}$ (a) and $D_{\text {it }}$ (b) for samples shown in Fig. 5. Open marks present $D_{\mathrm{r}}$ and $D_{\text {it }}$ for first laser or plasma treatment. Solid marks present $D_{\mathrm{r}}$ and $D_{\text {it }}$ for second laser treatment. Power intensities at second laser irradiation present in the figure.

$4.40 \times 10^{4} \mathrm{~W} / \mathrm{cm}^{2}$ laser irradiated sample. The high power laser heating made $D_{\mathrm{r}}$ much higher than $D_{\mathrm{it}}$. This indicates that high power laser induced defects are very sensitive to carrier recombination. In the case of laser irradiation $4.00 \times 10^{4} \mathrm{~W} / \mathrm{cm}^{2} \quad D_{\mathrm{r}}$ and $D_{\text {it }}$ were $1.8 \times 10^{11} \mathrm{~cm}^{-2}$ and $8.4 \times 10^{10} \mathrm{~cm}^{-2} \mathrm{eV}^{-1}$. Low $D_{\mathrm{r}}$ and $D_{\text {it }}$ were obtained to be $6.9 \times 10^{10} \mathrm{~cm}^{-2}$ and $1.8 \times 10^{10} \mathrm{~cm}^{-2} \mathrm{eV}^{-1}$ by two step laser irradiation at $4.0 \times 10^{4}$ followed by $3.70 \times 10^{4} \mathrm{~W} / \mathrm{cm}^{2}$, although they were kept high as $2.9 \times 10^{12} \mathrm{~cm}^{-2}$ and $4.2 \times 10^{11} \mathrm{~cm}^{-2} \mathrm{eV}^{-1}$ by two step laser irradiation at $4.40 \times 10^{4}$ followed by 
$3.70 \times 10^{4} \mathrm{~W} / \mathrm{cm}^{2} . D_{\mathrm{r}}$ and $D_{\text {it }}$ were increased to $1.9 \times 10^{12} \mathrm{~cm}^{-}$ 2 and $7.9 \times 10^{11} \mathrm{~cm}^{-2} \mathrm{eV}^{-1}$ by the plasma irradiation. Plasma induced defects were sensitive to carrier trap as well as carrier recombination. $D_{\mathrm{r}}$ and $D_{\text {it }}$ decreased to $8.8 \times 10^{9} \mathrm{~cm}^{-2}$ and $8.1 \times 10^{9} \mathrm{~cm}^{-2} \mathrm{eV}^{-1}$ by additional laser irradiation at $3.57 \times 10^{4} \mathrm{~W} / \mathrm{cm}^{2}$. Defect states caused by plasma irradiation were easily eliminated by laser irradiation, in contrast to the case of defect states caused by high power laser irradiation $4.40 \times 10^{4} \mathrm{~W} / \mathrm{cm}^{2}$, which were hardly cured by additional laser heating.

Via those experimental results and analyses, we interpret that the point defects such as lattice vacancies (point defects) are mainly generated in the case of plasma irradiation and laser rapid heating at a low power. The point defects play roles in carrier recombination and carrier trap. They are easily eliminated by subsequent laser heating because interstitial silicon atoms are moved back to the lattice sites by laser heating. When samples were heated with high power intensity at $4.40 \times 10^{4} \mathrm{~W} / \mathrm{cm}^{2}$, large scale defects such as dislocation loops or vacancy clusters were probably formed [14]. Many silicon atoms moved and this mass action probably forms the large scaled defects. Those defects would not be removed by additional laser heating. Crystallographic analysis is further necessary to make defect structure formed by high power laser irradiation clear.

\section{Summary}

We investigated laser-induced passivation silicon surface using 940-nm semiconductor laser heating. $12 \Omega \mathrm{cm} \mathrm{n-}$ type $500-\mu \mathrm{m}$-thick silicon substrates coated with $100-\mathrm{nm}$ thick thermally grown $\mathrm{SiO}_{2}$ layers were prepared. $\tau_{\text {eff }}$ (top) and $\tau_{\text {eff }}$ (rear) were decreased to $2.5 \times 10^{-6}$ and $1.4 \times 10^{-4} \mathrm{~s}$ by laser irradiation up to $4.46 \times 10^{4} \mathrm{~W} / \mathrm{cm}^{2}$ for $4 \mathrm{~ms}$. They were also decreased to $1.7 \times 10^{-5}$ and $1.4 \times 10^{-4} \mathrm{~s}$ by $13.56 \mathrm{MHz} \mathrm{RF}$ Ar plasma at $50 \mathrm{~W}$ and $1.0 \mathrm{~Pa}$ for $120 \mathrm{~s}$. The substantial carrier recombination defect states were formed by high power laser and Ar plasma irradiation.

Additional laser heating was carried out for investigating increase in $\tau_{\text {eff. }}$ For the Ar plasma irradiated sample, $\tau_{\text {eff }}$ (top) and $\tau_{\text {eff }}$ (rear) were increased to $1.7 \times 10^{-3} \mathrm{~s}$, which was almost the same level of the initial sample by laser irradiation at $3.57 \times 10^{4} \mathrm{~W} / \mathrm{cm}^{2}$. In the case of laser irradiation at $4.00 \times 10^{4} \mathrm{~W} / \mathrm{cm}^{2}, \tau_{\text {eff }}$ (top) and $\tau_{\text {eff }}$ (rear) were decreased to $1.7 \times 10^{-4}$ and $2.9 \times 10^{-4} \mathrm{~s}$. They were increased to $3.9 \times 10^{-4}$ and $5.2 \times 10^{-4} \mathrm{~s}$ by additional laser irradiation at $3.7 \times 10^{4}$ $\mathrm{W} / \mathrm{cm}^{2}$. Change in $\tau_{\text {eff }}$ was similar to the case of Ar plasma irradiation. However, when the initial sample was heated by laser at $4.40 \times 10^{4} \mathrm{~W} / \mathrm{cm}^{2}, \tau_{\text {eff }}$ (top) and $\tau_{\text {eff }}$ (rear) decreased to $1.1 \times 10^{-5}$ and $1.5 \times 10^{-4} \mathrm{~s}$. They were hardly changed by additional laser irradiation at $3.70 \times 10^{4} \mathrm{~W} / \mathrm{cm}^{2}$. Analysis of $\mathrm{C}-\mathrm{V}$ characteristics gave decrease in $D_{\text {it }}$ to $8.1 \times 10^{9} \mathrm{~cm}^{-2} \mathrm{eV}^{-1}$ in the case of laser heating of Ar plasma irradiated sample. Although $D_{\text {it }}$ was decreased to $1.8 \times 10^{10}$ $\mathrm{cm}^{-2} \mathrm{eV}^{-1}$ by second laser irradiation at $3.7 \times 10^{4} \mathrm{~W} / \mathrm{cm}^{2}$ of the sample first heated by laser irradiation at $4.00 \times 10^{4}$
$\mathrm{W} / \mathrm{cm}^{2}$, it kept a high value of $4.2 \times 10^{11} \mathrm{~cm}^{-2} \mathrm{eV}^{-1}$ in the case of second laser heating at $4.00 \times 10^{4} \mathrm{~W} / \mathrm{cm}^{2}$ of the sample first heated at $4.40 \times 10^{4} \mathrm{~W} / \mathrm{cm}^{2}$. Experimental results shows that laser heating is effective to decrease the densities of carrier recombination states and carrier traps caused by $\mathrm{Ar}$ plasma irradiation and laser heating at a low power of $4.00 \times 10^{4} \mathrm{~W} / \mathrm{cm}^{2}$. We interpret that lattice vacancies were generated by those processes, and that they are effectively eliminated by additional laser heating. On the other hand, additional laser heating did not decrease the density of defects caused by laser heating at a higher intensity of $4.40 \times 10^{4} \mathrm{~W} / \mathrm{cm}^{2}$. Defects were probably too large for rapid laser heating to eliminate them.

\section{Acknowledgments}

This work was partly supported by Grant-in-Aid for Science Research C (No. 25420282 and 23560360) from the Ministry of Education, Culture, Sports, Science and Technology of Japan, and Sameken Co., Ltd.

\section{References}

[1] E. I. Shtyrkov, I. B. Khaibullin, M. M. Zaripov, M. F. Galyatudinov, and R. M. Bayasitov: Sov. Phys. -Semicond. (enbl. Transl.) 9 (1975) 1309.

[2] D. H. Lowndes, G. H. Kirkpatrick, Jr., S. J. Pennycook, S. P. Withrow, and D. N. Mashburn: Appl. Phys. Lett. 48 (1986) 1389.

[3] T. F. Deutsch, J. C. C. Fan, C. W. Turner, R. L. Chapman, D. J. Ehrlich and R. M. Osgood, Jr.: Appl. Phys. Lett., 38 (1981) 144.

[4] T. Sameshima, S. Usui, and M. Sekiya: J. Appl. Phys. 62 (1987) 711.

[5] T. Sameshima, M. Maki, M. Takiuchi, N. Andoh, N. Sano, Y. Matsuda, and Y. Andoh: Jpn. J. Appl. Phys. 46 (2007) 6474.

[6] K. Ukawa, Y. Kanda, T. Sameshima, N. Sano, M. Naito, and N. Hamamoto: Jpn. J. Appl. Phys. 49 (2010) 076503.

[7] T. Sameshima, K. Betsuin, T. Mizuno and N. Sano: Jpn. J. Appl. Phys. 51 (2012)03CA04-1-6.

[8] M. Hasumi, J. Takenezawa, T. Nagao, and T. Sameshima: Jpn. J. Appl. Phys 50 (2011)03CA03-1-4.

[9] T. Sameshima, H. Hayasaka, and T. Haba: Jpn. J.Appl. Phys. 48 (2009) 021204-1.

[10]T. Sameshima, T. Nagao, S. Yoshidomi, K. Kogure, and M. Hasumi: Jpn. J. Appl. Phys 50 (2011)03CA02.

[11] A. S. Groove: Physics and Technology of Semiconductor Devices (Wiley, New York, 1967) Chap. 5.

[12] D. J. Fitgerald and A. S. Grove: Proc. IEEE, 54 (1966) 1601 .

[13] Y. Taur and T. Ning: Fundamental of Modern VLSI Physics (Cambridge University Press, Cambridge ,U. K. 1998) Chap. 2.

[14] S. S. Kapur, Dissertation of University of Pennsylvanisa, (Publicly accessible Penn Dissertations, 2010).109.

(Received: July 22, 2013, Accepted: April 19, 2014) 